Boletín de la Sociedad Geológica Mexicana

VOLUMEN 63, NÚM. 3, 2011, P. 463-477

\title{
Importancia de la hidrogeología urbana; ciencia clave para el desarrollo urbano sostenible
}

\author{
René Alberto Dávila Pórcel ${ }^{1, *}$, Héctor de León Gómez ${ }^{1}$ \\ ${ }^{1}$ Facultad de Ciencias de la Tierra, Universidad Autónoma de Nuevo León, Carretera a Cerro-Prieto km. 8, Ex - Hacienda de \\ Guadalupe, C.P. 67700, Linares, Nuevo León, México \\ *rene.alberto.davila@gmail.com
}

\begin{abstract}
Resumen
La expansión urbana resultado del continuo crecimiento poblacional, la creación de industrias de diversos tipos y la paralela concentración de humanos en espacios reducidos, requieren de abastecimiento de agua suficiente y de buena calidad. En muchas ciudades del orbe el agua subterránea es la fuente principal del suministro para la población. A su vez, las actividades antropogénicas vierten grandes volúmenes de aguas residuales y efluentes industriales. El uso de una gran variedad de hidrocarburos y productos químicos en los procesos industriales ocasiona contaminación del suelo y de las fuentes de abastecimiento de agua potable. La complicada interacción entre los sistemas de flujo de agua subterránea y las ciudades (infraestructura, procesos, actividades y desechos) requiere de estudios cada vez más complejos, motivo por el cual emerge una nueva rama científica denominada "hidrogeología urbana" (HU), que es considerada una especialidad de las ciencias hidrogeológicas. El presente trabajo proporciona una visión general de la HU que permite entender los aspectos conceptuales, su campo de aplicación y su manejo. Se presenta información referida a la interdependencia de las ciudades y el aprovechamiento del agua, se detallan los procesos y mecanismos que estudia esta nueva rama del conocimiento, así como sus efectos asociados al medio físico y a la calidad química del agua (procesos contaminantes). Se analizaron casos de estudio tipo de varias ciudades alrededor del mundo con el fin de tener un escenario amplio sobre los impactos ambientales y los avances teóricos y tecnológicos desarrollados en la búsqueda de soluciones eficientes. Se resaltan las directrices y los modelos de gestión sostenible del agua subterránea en los medios urbanos desarrollados con el objetivo de proteger el medio ambiente y la salud pública de los residentes urbanos por medio de un análisis integral.
\end{abstract}

Palabras clave: hidrogeología, ciudad, recarga, subsidencia, contaminación, gestión del agua.

\begin{abstract}
Urban expansion as a result of continuous population growth as well as the creation of a large variety of industries and the parallel high human population density require a sufficient supply of clean water. In many cities around the world groundwater is the main source of potable water. In turn, anthropogenic activities discharge large volumes of waste water and industrial effluents. The use of a variety of chemicals and hydrocarbons causes pollution of groundwater supplies and soils. The complex interaction between groundwater flow systems and cities (infrastructure, city process, activities and discharge) requires increasingly complex studies, which is why a new branch of science has emerged under the name of "urban hydrogeology", considered to be a specialization within the hydrogeological sciences. This paper provides a general vision of urban hydrogeology that allows understanding its conceptual aspects, its scope of application and management. The information presented refers to the interdependence of cities and groundwater use. The paper details the processes and mechanisms studied through this new branch of knowledge as well as their effects in relation to the physical and chemical properties of water quality (pollution processes). Case studies are discussed in order to have a variety of viewpoints on environmental impacts, theoretical development and technological advances developed in order to seek efficient solutions. We highlight guidelines and models for sustainable groundwater management in urban settings that have been developed with the aim
\end{abstract}


of protecting the environment and urban residents' public health through an integral analysis.

Keywords: hydrogeology, city, recharge, subsidence, pollution, water management.

\section{Introducción}

La conservación, protección y gestión del agua subterránea (AS) son necesidades importantes en la mayoría de las ciudades del mundo (Shanahan, 2009), motivo por el cual la Asociación Internacional de Hidrogeólogos (International Association of Hydrogeologists, IAH) creó la Comisión de Aguas Subterráneas en Áreas Urbanas en el año 1993 (Vázquez-Suñé et al., 2005), con el objetivo de investigar el comportamiento y la problemática del AS en las ciudades que inició en la década de los ochenta, época en la que se realizaron muchos estudios del impacto del medio urbano sobre la calidad y la cantidad de AS (Morris et al., 2006; Wolf et al., 2007).

En la actualidad, la tendencia mundial es que la población rural disminuya, permitiendo el rápido crecimiento de las áreas urbanas originado en la espectacular concentración poblacional en megaciudades (Custodio, 2004). La densidad poblacional muestra que lo más importante en el ambiente del agua en las ciudades son los seres humanos (Welty, 2009). El incremento poblacional y el desarrollo industrial en áreas urbanas resulta en problemas de calidad y cantidad de AS (Grischek et al., 1996). El deterioro de la calidad del AS en los acuíferos de zonas urbanas se convierte en un riesgo potencial para las infraestructuras y las cimentaciones urbanas (Al-Sefry y Sen, 2006). En la determinación del impacto en los acuíferos se debe revisar la información hidrogeológica, los procesos de recarga y la calidad hidrogeoquímica del AS (Grischek et al., 1996). Debido a los problemas enlistados y a la urgente necesidad de conocer los procesos y parámetros que intervienen en la explotación, uso, vertido, conservación y protección del AS, catalogada como recurso valioso en el medio urbano, emerge una nueva rama científica dentro de las ciencias hidrogeológicas denominada "hidrogeología urbana" (HU).

La HU investiga las condiciones hidrodinámicas del AS, la recarga, el uso y calidad del agua, así como el manejo, preservación y repercusiones de los acuíferos de áreas urbanizadas y en sus alrededores, orientada al abastecimiento de la población y sus áreas urbanas, industriales, comerciales, de riegos intensivos, de recreo y parques públicos (Custodio, 2004). La HU juega un rol preponderante en la solución de los problemas de demanda de agua potable, de contaminación, de subsidencia y colapso del terreno, de interacción del AS con la infraestructura urbana, de inestabilidad de taludes y encharcamientos persistentes (Llamas y Custodio, 2003).

Actualmente, los estudios de HU están motivados por la importante interacción entre las aguas subterráneas (ASs) y el desarrollo socioeconómico de las ciudades. La progresiva urbanización afecta la disponibilidad del agua así como su calidad, lo cual tiene importantes implicaciones sociales, ambientales, económicas y políticas (Vázquez-Suñé et al., 1999). A futuro, la HU desempeñará un papel importante en la provisión de agua, al desarrollar sistemas separados que permitan la reutilización directa y generalizada, creando así recursos hídricos propios y aliviando la enorme presión depredadora realizada por las grandes urbes sobre el territorio y sus recursos (Custodio, 2004).

En los países en desarrollo, la HU prioriza los términos económicos y ambientales. Estos países tienen una creciente y constante demanda de agua en sus ciudades donde las cuestiones espacio-temporales que deben analizarse son: a) ciclo del AS relacionado al desarrollo de la urbanización; b) oscilaciones de los niveles de AS interconectados a la actividad humana; c) contaminación de AS producida por la actividad antropogénica; y d) la distribución de infraestructura subterránea y su relación con los flujos de AS (Vázquez-Suñé et al., 2005).

Investigadores y precursores de esta nueva rama científica proponen que los objetivos de la HU son: "Brindar una visión integral de los procesos y mecanismos hidrogeológicos que estudia la hidrogeología urbana, analizando los efectos asociados a la explotación, sobreexplotación, calidad, cantidad y presencia de contaminantes en el agua subterránea" (Custodio, 2004) e "Identificar los factores más significativos en el ciclo hidrogeológico urbano, así como, desarrollar y aplicar metodologías para cuantificar y controlar estos factores" (Vázquez-Suñé et al., 2005).

\section{Objetivo}

Revisar y analizar los procesos, factores y avances preponderantes de la hidrogeología urbana que determinan las relaciones e interacciones entre la hidrología, los materiales geológicos, la urbanización, la recarga, el flujo subterráneo, la extracción, la calidad hidrogeoquímica, el uso y los efectos que produce el agua subterránea en la infraestructura urbana, así como los mecanismos de desecho y los procesos contaminantes que afectan la calidad del agua subterránea en urbes en crecimiento y con desarrollo acelerado.

\section{Desarrollo del tema}

3.1. Interdependencia de las aguas subterráneas y la urbanización 


\subsubsection{El desarrollo urbano y las aguas subterráneas}

El suministro de agua, saneamiento y drenaje son servicios claves en el proceso de urbanización, en el desarrollo económico y en la salud pública de los habitantes urbanos (Foster et al., 2001; Al-Sefry y Sen, 2006; Shen, 2008). En el medio urbano las AS son casi siempre el primer recurso que se utiliza con fines de abastecimiento de agua, porque poseen los siguientes beneficios: a) son de excelente calidad natural; b) son más seguras como fuente de abastecimiento en época de sequias; c) pueden utilizarse para el abastecimiento público y la utilización privada independientemente; y d) son atractivas en términos de inversiones de capital. Razones por las cuales contribuyen al desarrollo integral de las ciudades (Foster et al., 2001; Kuroda y Fukushi, 2008). La explotación de AS permite la expansión del sector industrial que soporta el crecimiento urbano, pero a su vez, se incrementa el vertido de productos secundarios indeseables que provienen de las diversas actividades urbanas. La disposición de las aguas servidas domésticas en los asentamientos residenciales con gran densidad pueden ser semipermanentes, especialmente en los distritos de bajos ingresos donde se usan tanques sépticos, pozos negros o letrinas para desechar las mismas (Foster et al., 2001). La mayor parte de la eliminación de desechos sólidos se hace mediante rellenos sanitarios o vertederos a cielo abierto que producen lixiviados que se infiltran en el terreno, los cuales pueden tener un grave impacto en la calidad del AS destinada al abastecimiento (Foster et al., 2001). Todos estos factores son la consecuencia del crecimiento poblacional, una realidad de los países en desarrollo donde las inmigraciones poblacionales inesperadas hacia los centros urbanos incrementan la presión sobre las ASs y los servicios municipales de una manera sin precedentes en la historia de la humanidad (Ribeiro, 2002).

\subsubsection{Evolución del proceso de urbanización}

Los países en desarrollo son los que presentan el mayor índice de crecimiento demográfico del mundo, sus tasas de crecimiento urbano y poblacional no tienen comparación en la historia humana. El surgimiento de asentamientos informales situados en tierras marginales o en distritos periurbanos crea una rápida expansión física. La proporción de personas de bajos recursos económicos oscila entre el 30 $\%$ y el $60 \%$ del total de la población urbana, donde el AS es un factor especialmente importante para el desarrollo urbano (Foster et al., 2001; Takizawa, 2008b). La evolución del proceso de urbanización en las ciudades contempla cuatro importantes etapas que son (Shen, 2008; Shanahan, 2009):

a) Ciudad preindustrial - usando ASs locales: Los residentes perforan pozos poco profundos para autoabastecerse de agua. Las aguas residuales se desechan en pozos negros y tanques sépticos en cada una de las residencias (Figura 1).

b) Ciudad industrial - disminución de los niveles de agua: Esta fase coincide con el desarrollo industrial y la demanda de agua crece. Paralelamente, la infraestructura de extracción de agua se consolida, los sistemas de suministro de agua potable y de alcantarillado son implementados. Posteriormente, este desarrollo provoca que los acuíferos experimenten importantes descensos del nivel freático (NF) hasta que los acuíferos deben ser controlados e intervenidos como se muestra en la Figura 2. Si los efectos de este descenso del NF debajo de la ciudad industrial son numerosos pueden ocasionar subsidencia de terreno (ST), la profundización de pozos debido al abatimiento del agua, el incremento en los costos de bombeo, la intrusión de agua salina (en el caso de ciudades costeras) y cambios en la capacidad portante de los suelos que afectan las cimentaciones de los edificios (Downing, 1993). Por estos efectos la ciudad se vuelve dependiente de fuentes distantes y la infraestructura de transporte de agua debe ser ampliada desde las afueras de la ciudad para proveer el líquido vital a sus habitantes.

c) Ciudad postindustrial - elevación del nivel del agua subterránea: En esta fase, se recuperan los niveles naturales del AS, se produce una transición a una economía postindustrial, se pierde el suministro local de agua debido a la contaminación industrial, se desarrolla el abastecimiento de fuentes distantes y seguras de agua (Figura 3). Las ASs ascendentes afectan las propiedades estructurales de los edificios, la capacidad de suelos de soportar carga, las mamposterías y el concreto, se presentan subpresiones hidrostáticas, encharcamientos y expansión de suelos, se infiltran en los alcantarillados poniendo en riesgo los sistemas de recolección de aguas servidas (Bassington, 1990; Johnson, 1994; Lerner, 2004).

d) La futura ciudad bajo el cambio climático y el crecimiento poblacional: El cambio climático afectará la cantidad y distribución de la recarga del AS, presentando intensas lluvias en regiones altas y tropicales y menos lluvia para las latitudes subtropicales (Solomon et al., 2007). Las ciudades del mundo necesitaran proveer mucha más agua y lidiar con mayores problemas de contaminación. En este contexto, el AS será más vulnerable y fuertemente explotada. La Figura 4 esquematiza el comportamiento del sistema bajo estas condiciones (Shanahan, 2009).

\subsection{Impactos causados por la explotación de agua subterránea}

Los principales impactos provocados por el aprovechamiento del AS de los acuíferos son: extracción excesiva, fluctuación del NF, subsidencias del terreno e intrusión de aguas salinas (Carbognin et al., 1984; Custodio, 2002).

\subsubsection{Extracción excesiva de acuiferos}

La explotación de agua subterránea (AS) en zonas urbanas depende de su disponibilidad en los acuíferos. El medio geológico es muy importante debido a que las formaciones geológicas transportan y almacenan AS; estas cualidades dependen de la litología, grado de 


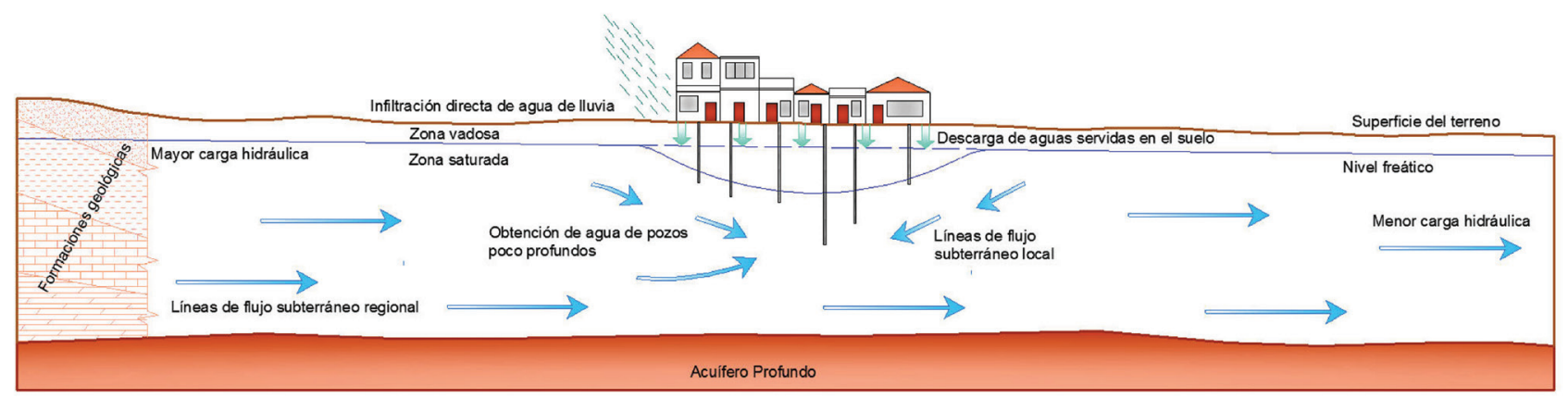

Figura 1. Esquema de la situación de una ciudad inicial (modificado de Foster et al., 2001; Lerner, 2004).

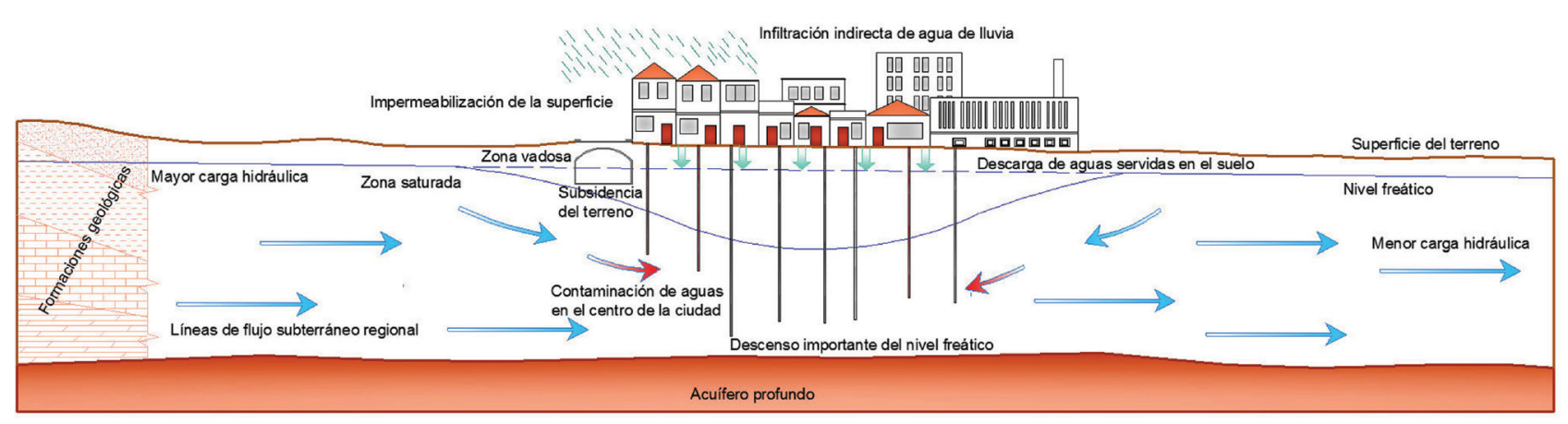

Figura 2. El sistema bajo el auge de una ciudad industrializada (modificado de Foster et al., 2001; Lerner, 2004).

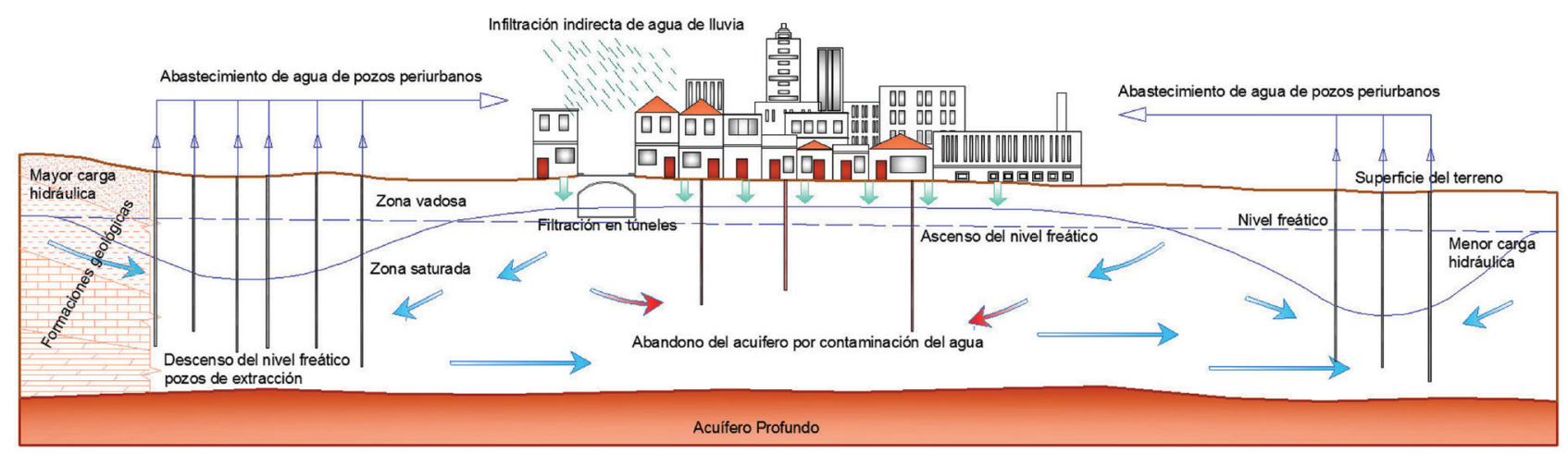

Figura 3. Ciudad postindustrial y su impacto sobre el agua subterránea (modificado de Foster et al., 2001; Lerner, 2004).

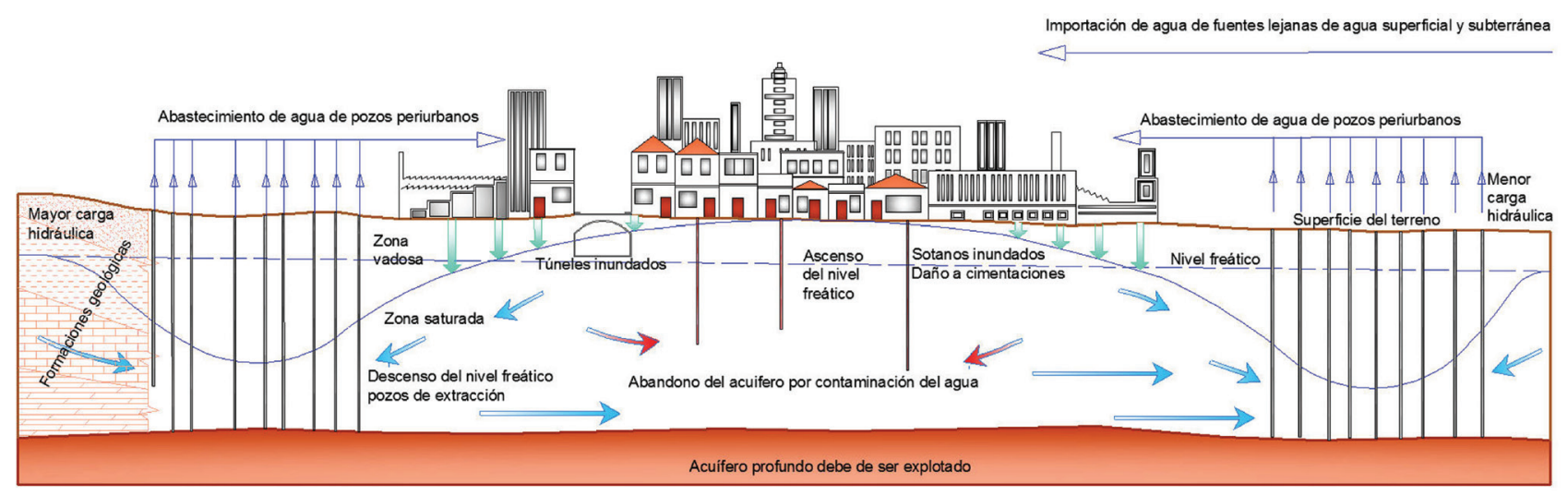

Figura 4. Ciudad del futuro bajo el cambio climático (modificado de Foster et al., 2001; Lerner, 2004). 
compactación y consolidación, distribución espacial del paquete estructural y grado de fracturamiento e intemperismo de las rocas que constituyen los acuíferos. En realidad la designación de extracción excesiva de AS de un acuífero es principalmente un adjetivo que trata de calificar a una evolución preocupante bajo determinados puntos de vista, sin que tenga una significación hidrodinámica precisa (Custodio, 2002; Kontogianni et al., 2007). La explotación y gestión de AS en el medio urbano solo es posible si las formaciones geológicas tienen una permeabilidad moderada o alta agrupándose en: a) sedimentos no consolidados, incluyendo sedimentos aluviales, que suelen contener grandes volúmenes de AS que permiten la extracción económica y b) formaciones consolidadas que cuando están fracturadas pueden ser muy permeables y contener grandes cantidades de agua (Foster et al., 2001). El mayor tema de preocupación en el estudio del AS ha sido la extracción excesiva (Drewes et al., 2003; Custodio, 2004), que puede ser definida como la situación en la que durante varios años la extracción media de AS de un acuífero supera o se aproxima a su recarga media (Custodio, 2002; CNA, 2007; Molina et al., 2009). Los impactos de la extracción excesiva de AS son: un descenso continuo del NF, menor volumen disponible de AS, deterioro de su calidad, incremento en el costo de extracción, daños ecológicos e ineficiencia en el manejo del AS y asentamientos diferenciales del terreno (O'Shea, 1993; Lerner y Barrett, 1996; Custodio, 2002; Shen, 2008).

\subsubsection{Fluctuaciones de los niveles del agua subterránea}

La extracción del agua subterránea (AS) provoca que el nivel freático (NF) descienda. Los pozos tienen a su alrededor la superficie freática la cual debido a la extracción forma un cono de depresión. Cuando muchos pozos están ubicados unos cerca de otros, sus conos de depresión se llegan a superponer, causando el descenso general del NF y cambiando la distribución de la carga hidráulica dentro del sistema acuífero (Foster et al., 2001; Hooker et al., 1999; Shanahan, 2009; Zhang et al., 2010). Las variaciones de los NFs en áreas urbanas dependen de tres factores principales que son: a) la transmisividad del acuífero (recarga vertical y el flujo horizontal); b) la heterogeneidad y anisotropía de los materiales que constituyen el acuífero; y c) las explotaciones continuas de AS provocando un déficit de recarga (Wilkinson, 1994; Hooker et al., 1999; Foster et al., 2001; Shanahan, 2009). Es lógico que durante los periodos de tiempo de descenso del NF, se favorezca la construcción de infraestructura urbana porque la excavación no tiene la necesidad de drenaje ni de protecciones impermeables. Posteriormente, cuando los niveles de extracción disminuyen o cesan, se produce el ascenso del NF; en esta situación se presentan serios problemas con las estructuras enterradas o excavadas, las cuales pueden quedar inundadas temporalmente $o$ permanentemente (Chilton, 1999). El ascenso de los NFs se produce debido a: fugas en las tuberías de suministro de agua potable (estimadas entre el $20 \%$ y el $30 \%$ del volumen total distribuido), filtración de pozos negros, recarga indirecta de la precipitación, exceso en la irrigación de jardines, fugas de tanques de almacenamiento en el subsuelo y fugas de los alcantarillados sanitarios. El incremento del contenido de humedad pone en riesgo de licuefacción al suelo saturado afectando a edificios e infraestructura urbana (Al-Sefry y Sen, 2006; Hayashi, 2008).

\subsubsection{Subsidencia del terreno}

La continua y excesiva extracción de AS puede llevar a la subsidencia del terreno (ST) que causa importantes pérdidas económicas (Ng et al., 2009). El AS sirve como elemento estructural en la subsuperficie, lo cual contribuye con la integridad geológica del terreno; la remoción del AS puede destruir esta integridad y llevar a la ST (Shanahan, 2009). Este problema se presenta en depósitos de sedimentos recientes arcillosos, a causa de la disminución de la presión intersticial o bien colapsos locales si hay cavidades kársticas o sifonamiento y arrastre de arenas. La ST puede ser de extensión regional cuando es causada por bombeo muy excesivo y continuo de AS y combinado con el peso de las construcciones civiles (Larson et al., 2001; LoboGuerrero Uscátegui, 2003; Carreón-Freyre et al., 2005; Ochoa-González y Carreón-Freyre, 2006; Abidin et al., 2008). La ST es gradual, repentina o con la presencia de grietas, que se deben al movimiento y deformación del material del subsuelo por extracción de petróleo, gas o descenso del AS. El incremento en la presión efectiva por la reducción del AS induce la compactación del subsuelo no consolidado en las capas del suelo, ocurriendo finalmente la subsidencia (Arzate-Flores et al., 2006; Kontogianni et al., 2007; Rahnama y Moafi, 2009). La ST puede ser monitoreada por varios métodos como son: extensómetros de nivelación (Bitelli et al., 2000), estaciones totales (Leakea y Mock, 1997), registros con GPS (Abidin et al., 2008; Bitelli et al., 2000) e interferometría de radares espaciales (Ferreti et al., 2000, 2001). Radares espaciales y aéreos (SyntheticAperture Radar, SAR) son las aplicaciones tecnológicas más efectivas usadas en muchos problemas de ingeniería y geología, los que permiten determinar la ST. La difracción persistente de interferometría de radar de apertura sintética, (DInSAR) puede registrar deformaciones de series de tiempo con exactitud de milímetros del nivel del terreno, estos resultados dependen de un gran número de imágenes de calidad SAR. Estas técnicas han sido aplicadas en diversos trabajos de monitoreo de desplazamientos superficiales y problemas geológicos, probando ser adecuadas para este tipo de monitoreo en áreas urbanas (Amelung et al., 1999; Declercq et al., 2005; Dehls et al., 2002; Kim et al., 2007; Kim, 2003; Massonnet y Feigl, 1998; Moon et al., 1998; Ng et al., 2009; Raymond y Rudant, 1997; Wright y Stow, 1999). El monitoreo de la ST es una tarea importante en protección civil y decisiones de política pública (Kim et al., 2007), así como la predicción de la ST es útil en la gestión del uso óptimo del AS (Rahnama y Moafi, 2009). 


\subsubsection{Intrusión de aguas salinas}

A medida que baja el nivel freático (NF) puede invertirse la dirección de flujo subterráneo y la interfase entre el acuífero y las aguas salinas lo cual permite el avance de aguas salobres tierra adentro. Este problema acontece en ciudades costeras donde la intrusión marina trae agua salada rica en sulfatos que en contacto con el concreto y acero de refuerzo de las cimentaciones y estructuras sepultadas acelera sus tasas de corrosión (Foster et al., 2001; VázquezSuñé et al., 2005). Una vez que la salinidad se ha difundido en el agua alojada en los poros de la matriz de grano fino del acuífero, su elución llevará décadas o siglos, incluso si se restablece un flujo de aguas dulces subterráneas hacia la costa (Foster et al., 2001).

\subsection{Calidad del agua y efecto de los contaminantes}

Aproximadamente el $80 \%$ de las enfermedades y de las muertes en los países en desarrollo están relacionados a aguas contaminadas (UNESCO, 2006). La composición química del agua subterránea (AS) puede ser identificada por las concentraciones y proporciones de sus elementos constituyentes (Al-Sefry y Sen, 2006; Lloyd y Heathcote, 1985). El valor del AS como recurso puede ser completamente o parcialmente perdido si es que el AS se encuentra contaminada (Shanahan, 2009). La contaminación del AS plantea serios problemas en áreas urbanas, los contaminantes pueden ser inorgánicos, orgánicos y microbiológicos (Kuroda y Fukushi, 2008). La contaminación proveniente de fuentes puntuales y de fuentes agrícolas es muy notoria. Las fuentes directas de contaminación pueden incluir: fugas de alcantarillas, de tanques sépticos, por escorrentía urbana, lixiviación de basurales y residuos sólidos, fugas de tanques de almacenamiento, de derrames de gasolina, de infiltración de ríos contaminados, de fertilizantes y pesticidas, uso de productos de deshielo y pozos abandonados sin protección. Las mezclas de aguas residuales con aguas cloradas para suministro pueden ocasionar contaminación por cloratos tóxicos y compuestos recalcitrantes (Vázquez-Suñé et al., 2005; Kuroda y Fukushi, 2008; Takizawa, 2008b). Las fugas de tanques sépticos, pozos negros y letrinas constituyen, probablemente, la fuente más común y propagada de contaminación difusa, cuya consecuencia inmediata es la migración de bacterias y virus patógenos a los acuíferos subyacentes (Foster et al., 2001; Kuroda y Fukushi, 2008). Gran número de industrias de textiles, metalurgia, mantenimiento de vehículos, lavanderías, imprentas, curtiembres y revelado de fotografías tienden a estar en zonas urbanas y generan efluentes como por ejemplo: lubricantes usados, líquidos ricos en metales, solventes y desinfectantes, que a menudo se descargan directamente en el suelo por derramamientos accidentales que causan problemas graves de contaminación de las ASs (Hooker et al., 1999; Foster et al., 2001).

El desplazamiento de áreas industriales o de riego intensivo que usan AS a causa del incremento de los precios del suelo y la presión por construir viviendas con un entorno más urbano y agradable reubica los focos de contaminación (Bucich y Nagy, 2003). La calidad del AS es normalmente peor bajo las ciudades que bajo las áreas rurales. Para lograr la gestión global del impacto urbano en la calidad del AS es necesario obtener los valores de fondo en condiciones naturales, posteriormente se debe incluir aspectos como la cuantificación del ingreso de contaminantes, la vulnerabilidad del acuífero, el nivel de contaminación del AS, la movilidad, degradación y toxicidad de los contaminantes, las direcciones y velocidad del flujo y el uso del AS con el objetivo de evaluar el riesgo contaminante de los procesos antropogénicos (Toussaint, 1994; Grischek et al., 1996).

\subsubsection{Fertilizantes y pesticidas}

Tanto el agua subterránea como el agua superficial están bajo serio riesgo de contaminación debido al excesivo uso de fertilizantes y pesticidas en la agricultura que mejoran la producción agrícola, debido al acelerado crecimiento poblacional y el desarrollo tecnológico alrededor del mundo la utilización de estos productos es ampliamente difundida generando contaminación en muchos países del mundo (Ekemen-Keskin, 2010). A veces, los contenidos de nitratos son menores en las áreas urbanas debido al uso extendido de fertilizantes en áreas intensamente cultivadas (Takizawa, 2008b). La contaminación por nitrato en las ciudades tiene fuentes no puntuales, por lo que la correlación exacta con el uso de suelo no es posible. La presencia de sulfatos puede deberse a la posible adición de emisiones industriales y fugas de los alcantarillados urbanos (Burston et al., 1993; Ford y Tellam, 1994; Lerner et al., 1994). Otra actividad contaminante es el abandono de pozos y obras de captación de AS debido a la infiltración de agua de mala calidad, a la presencia de microorganismos, amonio, nitritos y un alto contenido de nitratos y aumento de la dureza y salinidad del AS (Apodaca et al., 2002).

\subsubsection{Aguas servidas}

Las posibles fuentes urbanas de contaminación por nitratos son: industrias, alcantarillados y zonas de recreación (Foster et al., 2001). La contaminación por nitratos es el tipo más prevalente de contaminación antropogénica y está fuertemente relacionada al patrón del uso de suelo (Ator y Denis, 1997; Elhatip et al., 2003; Kuroda y Fukushi, 2008; Rajmohan et al., 2009). La contaminación debida a fugas de los sistemas de alcantarillado en las ciudades es reconocida como un gran problema (Short, 1988; Lerner y Barrett, 1996). En acuíferos kársticos muy permeables el vertido directo de aguas servidas por medio de tanques sépticos, letrinas y pozos negros produce mayor contaminación en los pozos someros que en los pozos profundos, pero estos últimos también serán seriamente afectados con el paso del tiempo (Foster et al., 2001). La ausencia de alcantarillado entubado para la recolección de efluentes domésticos, 
industriales y drenaje pluvial causa el vertido de las mismas en el suelo, por esta razón se produce el deterioro de los acuíferos con concentraciones elevadas de nitrato y cloruro bajo distritos densamente poblados (Foster et al., 2001; Takizawa, 2008b). Por otra parte, altas concentraciones de nitrato, cloruro y sulfato en las ASs son evidencia de contaminación proveniente de la agricultura (Nagarajan et al., 2010).

\subsubsection{Compuestos orgánicos volátiles (VOCs) e hidrocarburos y sus derivados}

La contaminación por VOCs fue detectada en varios lugares industrializados, también se pueden presentar productos petrolíferos fugados de tanques de almacenamiento de gasolineras y domicilios, lavado de las superficies de calles y vertidos accidentales irregulares (Panchow y Cherry, 1996; Drewes et al., 2003; Custodio, 2004; Kuroda y Fukushi, 2008). Se pueden encontrar disolventes de hidrocarburos clorados (CHSs) como el Tricloroetileno (TCE), Tricloroetano y Triclorometano, Tetracloroetileno (PCT) y varios tipos de derivados de petróleo. Se reportaron varios incidentes de derrames de derivados de petróleo, en donde varios de estos contaminantes pueden disolverse en el AS, los contaminantes orgánicos son peligrosos para la salud de las personas y el medio ambiente(Schellekens, 1974; Schwille, 1975; Vanlooke et al., 1975; Kessler y Rubin, 1987; Schirmer y Butler, 2004). Debido a su amplio uso, movilidad y persistencia, los compuestos orgánicos volátiles clorados son considerados los de más alto riesgo de todos los contaminantes industriales (Ellis y Rivett, 2007).

\subsubsection{Metales Pesados y compuestos especiales}

Los principales metales y compuestos químicos presentes en el agua subterránea (AS) son: iones mayores $\mathrm{Na} \mathrm{Mg}, \mathrm{Ca}, \mathrm{K}, \mathrm{Cl}$ y $\mathrm{SO}_{4}$; contaminantes de nitrógeno como $\mathrm{NH}_{4}, \mathrm{NO}_{2}, \mathrm{NO}_{3}$; y elementos traza como As, $\mathrm{Cd}, \mathrm{Cr}$, $\mathrm{Cu}, \mathrm{Hg}, \mathrm{Mo}, \mathrm{Ni}, \mathrm{Pb}, \mathrm{Se}, \mathrm{U}, \mathrm{V}, \mathrm{Zn}, \mathrm{Fe}, \mathrm{Mn}, \mathrm{Br}, \mathrm{Cs}, \mathrm{Co}, \mathrm{F}$, Sn, Sr, Te, Ba, B y S (Fuge, 2004; Krishna y Govil, 2004; McBride, 2004; Atabey, 2005; Danis, 2005; He et al., 2005; Nouri et al., 2006; Guo et al., 2007; Kumbur et al., 2008). La mayor fuente de contaminación por metales pesados en el suelo son los fertilizantes orgánicos (Mikayilov y Acar, 1998). Los fertilizantes para jardines tienen efectos relacionados a altos contenidos de $\mathrm{Hg}, \mathrm{Pb}, \mathrm{Zn}$ y Cu (Manta et al., 2002). El fosforo está presente en la mayoría de los fertilizantes, el uso de bio-sólidos y composta incrementa las cantidades de $\mathrm{Cu}, \mathrm{Zn}, \mathrm{Pb}, \mathrm{Cd}$, Fe y Mn en los suelos. La movilidad y disponibilidad de los elementos traza están controlados por varios procesos químicos y bioquímicos, como la precipitación, disolución, adsorción-desorción y óxido-reducción. Todos estos procesos están afectados por el $\mathrm{pH}$ y los procesos biológicos del suelo y del AS (He et al., 2005). La diversidad derivada de los fertilizantes incluye $\mathrm{Na}, \mathrm{Mg}, \mathrm{Cl}$ y $\mathrm{SO}_{4}$, además de la interacción agua-roca. $\mathrm{El}$ monitoreo espacial del $\mathrm{NO}_{3}$ es de crucial importancia para evaluar la calidad del $\mathrm{AS}$, debido a que el $\mathrm{NO}_{3}$ tiene correlaciones positivas con metales pesados (EkemenKeskin, 2010). La presencia de $\mathrm{Pb}, \mathrm{Cr}, \mathrm{Cu}, \mathrm{Zn}, \mathrm{S}$ y V se debe a la contaminación del suelo debido a la industrialización y actividades agrícolas intensas (Krishna y Govil, 2004). La industria es la responsable de introducir metales pesados al AS hecho que se verificó por la correlación de uso de suelo y presencia de metales (Burston et al., 1993; Ford y Tellam, 1994; Lerner et al., 1994; Takizawa, 2008b). La presencia de disolventes orgánicos y el resultado del amplio uso de detergentes y substancias similares y de componentes de boro se convierten en serios riesgos de contaminación de los recursos hídricos (Stenger et al., 2006; Kuroda y Fukushi, 2008).

\subsection{Problemática ambiental del agua subterránea urbana}

La extracción excesiva y contaminación del AS se ha convertido en el mayor problema ambiental en la mayoría de ciudades de países industrializados y en desarrollo. Los países con mayor escasez de agua son: China, India, México, España, Egipto, Kazajstán, Irán, Australia y Pakistán, que después de muchos años de explotación inmoderada de los acuíferos enfrentan problemas con sus sistemas de manejo del agua urbana como el descenso del nivel freático, contaminación de AS, subsidencia del terreno e intrusión de agua de mar (en ciudades costeras). Mejorar el manejo de los recursos de AS es una necesidad urgente para mitigar los problemas ambientales, motivo por el cual un enfoque integral para la mejor gestión del agua debe incluir mejoras tecnológicas, innovaciones legislativas y manejo ambiental en las ciudades de los países en desarrollo (Shen, 2008).La gestión de las ASs representa un desafío político especial, porque el público y los tomadores de decisiones conocen muy poco acerca del estado del AS como recurso.

Los cambios negativos en la calidad y cantidad del AS pueden producirse durante años o décadas sin acción o noticia alguna. Por tanto, es necesario incluir herramientas de manejo, monitoreo, difusión y regulación en las políticas relacionadas a la gestión del AS (Shanahan, 2009; Takizawa, 2008b). El tratamiento y eliminación de la aguas servidas domésticas en ciudades florecientes de los países en desarrollo es una prioridad para proteger el AS urbana (Takizawa, 2008b). Los impactos también se presentan en la salud pública de los ciudadanos, por ejemplo, las ASs con valores altos de sólidos totales disueltos (STD) no son adecuados para consumo humano y riego (Fetter, 2001; Freeze y Cherry, 1979). Así mismo, las aguas residuales de las fugas del alcantarillado son la mayor fuente contaminante con un impacto negativo en el AS (Reynolds y Barrett, 2003; Foster y Chilton, 2004; Morris et al., 2006).

Las cuencas hidrológicas son integradores naturales de cursos de agua, que pueden transportar contaminantes y otros impactos humanos por medio de las AS por lo que la gestión del AS debe incluir permisos de bombeo, definición de usos permitidos, delineación de áreas de protección, identificación de contaminantes y monitoreo entre otras 
actividades (Ames, 2002). La disponibilidad de AS debe incluirse en la planificación de los recursos hidráulicos con el fin de lograr la sostenibilidad ambiental en base al estudio de: a) cuantificación de los volúmenes de ASs; b) usos posibles; c) mejoras técnicas en la construcción de pozos de extracción d) estudios de viabilidad económica; e) propuestas de manejo; f) sistemas de control del nivel piezométrico y g) la explotación del acuífero (VázquezSuñé et al., 1999).

\section{Perspectivas futuras de la hidrogeología urbana}

Actualmente está reconocido que la empresa más importante del desarrollo sostenible es la gestión y la toma de decisiones (Kropp, 2010). Es por eso que la tendencia actual es enfocar los problemas desde un punto de vista integral, tratando los problemas de manera conjunta. Para analizar este macro problema se propusieron modelos de gestión integral del agua en ambientes urbanos que aplican diferentes metodologías, como por ejemplo: MUSIC (Wong et al., 2002) y PURRS (Kuczera y Coombes, 2002), destinados a la gestión de aguas pluviales; los sistemas de información geográfica son una tecnología importante que permite modelar y transferir información de diversa índole y desarrollar modelos como el ICS (Clifforde et al., 1999), que simula el comportamiento de aguas servidas y que puede ser integrado con otros modelos. Por otra parte, el modelo Aquacycle (Mitchell et al., 2003; Lekkas et al., 2008) puede modelar el reúso de aguas pluviales y servidas basado en un balance de la cantidad de agua. Hoy en día, el modelo UVQ (UrbanVolume and Quality) es el único modelo disponible que puede considerar la simulación conjunta de aguas pluviales, aguas residuales, abastecimiento de agua, agua subterránea y presencia de contaminantes desde un enfoque integral (Wolf et al., 2006).

\section{Casos de estudio de hidrogeología urbana}

\subsection{Ciudades alrededor del orbe}

Algunas ciudades han sufrido depresiones de AS significativas de entre 20 y $50 \mathrm{~m}$, como es el caso de Bangkok (Tailandia), Manila (Filipinas) y Tianjin (China), entre otras; así como descensos del NF de entre 10 y 20 m como Beijing, Shanghai y Xian (China) y Madrás (India). En todos esos casos, ha habido un deterioro de la calidad de aguas subterráneas y/o asentamiento del terreno a raíz de la caída de los niveles. Actualmente, alrededor de 45 ciudades de China están experimentando algún asentamiento, lo que indica que la caída de los niveles freáticos es generalizada (Foster et al., 2001).

Las subsidencias del terreno debidas al descenso de AS se presentaron en muchas regiones del mundo, como Shanghai y Su-Xi-Chang (China); Taipei (Taiwan); Cheshire
(Gran Bretaña); Venecia (Italia); Ciudad de México, Morelia, Celaya, Querétaro y Aguascalientes (México); Bangkok (Tailandia); Valle de San Joaquín y Valle de Santa Clara (California, USA); Wariake (Nueva Zelanda); North West (Sudáfrica); el valle de Latrobe (Australia); Rafsanjan (Irán); Brisbane, Camberra, Sydney, Newcastle y Perth (Australia); Busan (Corea); Yakarta (Indonesia); Tesalia (Grecia); y Tokio y Osaka (Japón) (Lofgren, 1969; Gambolati y Frezze, 1973; Poland, 1982; Adrian et al., 1999; Zhang y Wei, 2005; Ávila-Olivera y Garduño-Monroy, 2006; Phien-wej et al., 2006; Kim et al., 2007; Kontogianni et al., 2007; Abidin et al., 2008; Aichi, 2008; Takizawa, 2008a; Ng et al., 2009; Rahnama y Moafi, 2009; Zhang et al., 2010).

\subsubsection{Dresden - Alemania}

Cerca de $80 \%$ de las aguas subterráneas se encuentran contaminadas, presentando valores elevados de sulfato, boro, halógenos, orgánicos absorbibles (AOX) y halógenos volátiles orgánicos (VOC), por lo que la temperatura del agua subterránea (AS) bajo las áreas urbanas tiene 2 a $3{ }^{\circ} \mathrm{C}$ más que las zonas rurales. Los valores de conductividad eléctrica exceden los $1000 \mu \mathrm{S} / \mathrm{cm}$, los cuales son correlacionables con concentraciones altas de sulfatos y nitratos. Las concentraciones de sulfatos varían grandemente en el intervalo de 100-600 mg/L (Grischek et al., 1996). Adicionalmente, se encontró la presencia de tetracloroetileno (TCE) en las capas superiores del acuífero cuaternario, donde la principal fuente de contaminación son las limpiezas en seco, fábricas de instrumentos eléctricos, maquinaria, lubricantes y plásticos, otras potenciales fuentes pueden ser talleres de reparación de automóviles, forrajeras y hospitales. La biodegradación de percloroetileno (PCE) no se produce en ambientes aeróbicos, pero el TCE si puede ser deshalogenado en condiciones aeróbicas. Una disminución de la contaminación del AS puede ser lograda por procesos de remediación. Finalmente, procesos intensos de remediación de más de 10 años pueden lograr mejoras importantes en la calidad del AS (Cook et al., 1988; Grischek et al., 1996; Toussaint, 1994).

\subsubsection{Brighton, Liverpool, Londres, Birmingham y Coventry - Inglaterra}

En Brighton se produjo una intrusión salina, pero está pobremente documentada. En Liverpool, la extracción excesiva del acuífero fue provocada por la industria emplazada en la zona de Merseyside, donde el nivel freático (NF) bajó hasta inducir la reversión en la dirección del gradiente hidráulico produciendo intrusión de agua marina. Actualmente el ascenso del NF provoca inundaciones en los túneles del metro. En Londres la recarga del acuífero se produce en la periferia de la ciudad y el flujo subterráneo descarga en la ciudad, ocasionando problemas en las cimentaciones de las estructuras civiles y túneles. Esta situación se debe al ascenso del NF con contenidos elevados de sulfatos como producto de la oxidación de la pirita, calidad 
de agua que puede corroer el concreto. En Birmingham se produjeron ascensos y descensos del NF durante los 70; como posible solución se discutió la necesidad de explotar el AS para preservar la infraestructura urbana (Lerner y Barrett, 1996; O'Shea, 1993). Se detectó también la presencia de hidrocarburos, predominando los disolventes de hidrocarburos clorados (CHS) como el tricloroetileno (TCE), tricloroetano y el triclorometano, determinándose que existe una buena correlación entre el uso de suelo y los contaminantes implicando una contaminación del AS y del suelo. En Coventry se concluyó que todas las industrias que usan CHS causan contaminación. La aplicación de un modelo del flujo subterráneo permitió conocer las posibles plumas de contaminación considerando cada industria como fuente de contaminación (Lerner y Barrett, 1996).

\subsubsection{Yakarta - Indonesia}

Con 8.2 millones de habitantes, se ubica en tierras bajas deltaicas. La extracción excesiva del acuífero confinado con fines industriales ha producido el descenso del NF, como consecuencia se produjo una grave intrusión salina de agua de mar originada por la inversión del régimen de flujo subterráneo (Abidin et al., 2008) y subsidencia del terreno (de 3 a $6 \mathrm{~cm} /$ año), lo cual incrementó el riesgo de inundaciones por la marea en la parte norte de la ciudad. La restricción municipal de extracción de AS solo reduce la capacidad de satisfacer la demanda y alentar la extracción privada sin control. Yakarta tiene más de 900000 tanques sépticos, el efluente se descarga en canales superficiales debido a sumideros insuficientes y sobrecargados y a deficiencias en el mantenimiento, esto causa una contaminación grave de las aguas superficiales y plantea riesgos importantes para la salud pública. Se deben priorizar acciones para reducir la carga de contaminantes en el suelo; deben apreciarse mejor las amenazas de contaminación del AS y el impacto futuro de la calidad del agua para usuarios públicos y privados (Foster et al., 2001).

\subsubsection{Bangkok - Tailandia}

Se ha producido una depresión importante del NF que llegaba a los $60 \mathrm{~m}$ a mediados de los años 80 , ocasionando subsidencia del terreno y un aumento en la salinidad del AS. La adopción de medidas como la restricción del bombeo o la adaptación de la calidad del agua bombeada al uso final resultan muy difíciles cuando se debe controlar un gran número de usuarios en una economía con un alto espíritu empresarial, razones por las cuales es necesario mejorar la eficiencia del uso industrial y doméstico para reducir la extracción de AS (Foster et al., 2001).

\subsubsection{Tianjin - China}

Es la tercera zona urbana de China. El acuífero superior es salobre y está separado por una formación de arcillas de los acuíferos de agua dulce subyacentes. La extracción de AS de la ciudad se redujo al $25 \%$ del máximo alcanzado en 1982 a través de la gestión de la demanda y la creación de fuentes de suministro alternas, reduciendo de esta manera la subsidencia del terreno entre 10 y $20 \mathrm{~mm} / \mathrm{a}$. La gestión de la demanda de AS ha tenido logros significativos con un uso doméstico bajo igual a $110 \mathrm{~L} /$ día y el reciclaje industrial de agua llega al $75 \%$ del volumen total utilizado. Mediante medidas no fiscales (economía dirigida) se limitó con éxito la demanda urbana. Es preciso mejorar la eficiencia del riego agrícola para reducir la extracción total y se deben distribuir los puntos de extracción de una manera más uniforme para reducir las diferencias de carga hidráulica entre los acuíferos de agua salobre y dulce (Foster et al., 2001). Para conocer mejor la situación hídrica de la urbe, se aplicó un modelo de evaluación integral de beneficios (IBAM, por sus siglas en inglés), establecido en las bases de un proceso analítico jerárquico (AHP, por sus siglas en inglés), donde se evaluaron variables como: economía, intereses ambientales y sociedad. Los resultados muestran que se debe fortalecer el reciclamiento de las aguas residuales como actividad prioritaria (Xiaoqin, 2009).

\subsection{Ciudades de México}

\subsubsection{Ciudad de México}

En la Ciudad de México los niveles de los acuíferos de los sistemas entre montañas situados por debajo de la ciudad bajaron entre 5 y $10 \mathrm{~m}$ entre 1986 y 1992 (Foster $e t$ al., 2001). La Ciudad de México fue analizada por medio de interferometría de radar de apertura sintética (InSAR). Los resultados indican que la principal condicionante del peligro potencial de fracturamiento y daño a la infraestructura urbana y viviendas es el gradiente de subsidencia (CabralCano y Díaz-Molina, 2006).

\subsubsection{Morelia}

Se estudió la subsidencia del terreno (ST) por medio de interferometría diferencial de imágenes de radar de apertura sintética (DinSAR), que permite calcular el patrón de la interferencia (franjas) ocasionado por la diferencia de fase entre dos imágenes de la misma zona tomadas por un radar de apertura sintética en instantes diversos. Esta información se integró en un SIG, obteniéndose mapas de desplazamiento del terreno (Ávila-Olivera et al., 2006). Se realizaron mediciones periódicas de los niveles estáticos del agua subterránea (AS), demostrándose un abatimiento de $2.46 \mathrm{~m} / \mathrm{año}$, que disminuye la presión de poro del agua de los estratos, lo que ocasiona la subsidencia (Ávila-Olivera y Garduño-Monroy, 2006). También se utilizó la prospección no destructiva con Georadar, para detectar anomalías del subsuelo (Ávila-Olivera et al., 2006).

\subsubsection{San Luis Potosí}

Ciudad que presenta agrietamientos provocados por la continua extracción del AS. Se usaron métodos gravimétricos para determinar paleocanales, los cuales demostraron influir en el fallamiento del suelo (ArzateFlores et al., 2006). En el cálculo de los hundimientos se 
consideró la suma de los desplazamientos verticales de los estratos saturado y parcialmente saturado, que dependen del módulo de elasticidad promedio del estrato, del peso propio de la fase sólida del suelo, del peso del agua, del peso de la masa de suelo parcialmente saturada y de la relación de vacíos, los resultados evidencian que las zonas de agrietamientos coincide con la zona de máximos hundimientos (Pacheco-Martínez et al., 2006). Por otra parte, con el objetivo de enfrentar el problema de manera integral, se aplicó un modelo de balance total de agua en zonas urbanas llamado "Calidad y Volumen Urbano" (UVQ, por sus siglas en inglés), donde se generaron cuatro escenarios. Los resultados permiten evaluar el impacto de métodos alternativos en el ciclo total del agua, evidenciándose una fuerte dependencia del agua extraída del acuífero profundo. Las fugas del sistema de distribución de agua potable son la fuente más grande de infiltración en el acuífero más somero. El modelo aplicado es el más versátil y completo en la actualidad (Martínez et al., 2011).

\subsubsection{Querétaro}

Con 700000 habitantes, el estrangulamiento del acuífero produjo que el NF baje más de $100 \mathrm{~m}$ en promedio, la caída constante de los NF es de $3.5 \mathrm{~m} /$ año. La ST varía entre 0.4 y $0.8 \mathrm{~m}$. Los edificios e infraestructura urbana han sufrido daños considerables, por lo cual se estableció el plan decenal de estabilización del acuífero que aplica las siguientes medidas: a) reducción de fugas en tuberías; b) gestión de la demanda en el público; c) financiamiento de mejoras tecnológicas del riego y la eficiencia en el uso de agua; d) suministro de aguas servidas con tratamiento secundario a cambio de derechos de agua de pozo para el riego en las zonas periurbanas (Foster et al., 2001).

\subsubsection{Mérida}

El abastecimiento de agua potable de esta región es obtenido principalmente de un campo de pozos pertenecientes al acuífero kárstico, ubicado en los límites urbanos de la ciudad, el cual es vulnerable a la contaminación producto de las actividades de los más de 600000 habitantes. Sin embargo, para cubrir y asegurar la demanda de agua potable, se han realizado estudios hidrogeológicos en la porción sureste de Mérida para determinar la primera zona de reserva hidrogeológica (HRZ). Dicha zona se localiza por arriba de la ciudad de Mérida, con cero actividad industrial y rodeada de dos reservas naturales, así como una serie de cenotes. El único inconveniente es que en el área delimitada como HZR se localiza un antiguo relleno sanitario, el cual deberá ser removido y posteriormente conocer la calidad del agua subterránea presente en dicha zona. El agua es conducida a través de un acueducto hacia Mérida, en donde el gradiente hidráulico es controlado permanentemente, cuya finalidad es la de observar alguna inversión del mismo y que pueda causar contaminación a la HZR. De esta manera se busca obtener nuevas estrategias necesarias a corto y largo plazo para asegurar el abastecimiento de agua considerando los aspectos de protección ambiental, hidrogeológicos y control de la contaminación (Escolero et al., 2000, 2002). Contaminación por nitratos se obtuvo en estudios realizados por Pacheco et al. (2001) durante el período 1992-1993 en el campo de 12 pozos localizados entre las áreas de Muna y Tzucacab, al sur del estado de Yucatán. Las concentraciones de nitratos varía entre 7 y $156 \mathrm{mg} / \mathrm{L}$, en donde los pozos Chacsinkin y Peto sobrepasan 3.5 veces el límite máximo permisible para usos potables $(45 \mathrm{mg} / \mathrm{L})$. La fuente potencial de contaminación del agua subterránea, se le atribuye al uso de pesticidas y fertilizantes nitrogenados en actividades intensas de agricultura presentes en el área de estudio (Pacheco et al., 2001). Finalmente, se determinó que los pozos de abastecimiento se encuentran contaminados con solventes clorados (Foster et al., 2001).

\subsubsection{La Paz}

Sufrió extracción intensiva de agua subterránea durante los últimos años, lo que ha provocado la intrusión de agua de mar hacia el acuífero afectando las fuentes de abastecimiento de agua potable de la ciudad. Se reinterpretó 19 pruebas de bombeo como datos base para el modelo realizado con software HST3D con interface ARGUS ONE para resolver la ecuación de flujo en medio poroso, de transporte de soluto y la ecuación de densidad de variable. Se realizó un modelo de flujo y de transporte de solutos calibrado para el período 1990-2000 y utilizado para generar escenarios entre 2000-2010. Se simularon seis escenarios: status quo; relocalización de pozos; reasignación de volúmenes en pozos de agua potable; intercambio con agua residual; condiciones de equilibrio e inercial. Se concluye que solamente la combinación adecuada de los escenarios propuestos podría incidir positivamente en la reducción de la intrusión de agua de mar y proteger las aguas subterráneas del acuífero (Escolero y Torres-Onofre, 2007).

\subsubsection{León-Guanajuato}

Se reportó que el NF bajó 90 m entre 1960 y 1990, y entre 1 y 5 m por año entre 1990-1995, debido a la excesiva extracción de agua y a la especial configuración del subsuelo bajo la ciudad (Foster et al., 2001).

\subsubsection{Monterrey}

Con más de 4.3 millones de habitantes (INEGI, 2005) es una ciudad con actividades principalmente industriales, se ha caracterizado por tener un crecimiento demográfico acelerado, con climas extremos tanto en verano $\left(>50^{\circ} \mathrm{C}\right)$ como en invierno $\left(-12^{\circ} \mathrm{C}\right)$ y sequías prolongadas (período 1980-1981). La explotación excesiva y contaminación de los acuíferos de poros y grietas de la zona urbana como resultado de las actividades domésticas, municipales e industriales, aunado a la reducida capacidad de infiltración de las aguas superficiales en las áreas pavimentadas con concreto y asfalto que reducen los procesos de recarga del acuífero de Pozos Monterrey, afectando el almacenamiento de AS para el deficiente sistema de abastecimiento de agua 
potable compuesto de fuentes superficiales (presas $\mathrm{La}$ Boca, Cerro Prieto y El Cuchillo) y subterráneas (acuífero Buenos Aires, socavón de San Francisco, túneles de la Cola de Caballo, pozos de Mina y el citado acuífero de pozos Monterrey; de León-Gómez et al., 1994). Hasta la fecha existe un déficit de suministro de agua potable de más de $2 \mathrm{~m}^{3} / \mathrm{s}$, volumen necesario para cubrir la demanda $\mathrm{y}$ asegurar una calidad de vida digna de los regiomontanos (CAPDM, 1990).

\subsubsection{Linares}

La ciudad más importante de la región citrícola del estado de Nuevo León, se caracteriza por tener problemas de contaminación del acuífero de poros y grietas generados por desechos industriales hacia el río Camacho. La presencia de tiraderos y del relleno sanitario, los derrames de estaciones de servicio de gasolineras, la planta de tratamiento de aguas residuales, los desechos domésticos, los talleres mecánicos, la industria alimenticia, entre otros generan contaminación en el río Pablillo, colector principal de la presa Cerro Prieto que abastece de agua potable a la zona metropolitana de Monterrey.

\section{Discusión}

El desarrollo de las áreas urbanas afecta significativamente el ciclo hidrológico natural, el principal factor de cambio es el proceso de urbanización que evoluciona en varias etapas en el desarrollo de una ciudad. Cada etapa afecta de manera particular y significativa a los acuíferos. El proceso de recarga se modifica principalmente por la impermeabilización de la superficie, debido a la urbanización, produciéndose una recarga indirecta diferente a la recarga en zonas rurales. Actualmente, se tiene menos experiencia sobre la recarga en áreas urbanas y la existencia de diferentes fuentes de recarga como ser: infiltración en grietas, sumideros de agua pluvial, filtración de pozos negros, estanques, letrinas y red de alcantarillado y fugas del sistema de suministro, complican el escenario para determinar un volumen aproximado a la realidad.

La cuantificación detallada de los procesos de infiltración en áreas urbanas es imposible debido a la falta de datos, la gran variabilidad y la extrema no linealidad de las propiedades hidráulicas de los sedimentos en la zona no saturada. La aplicabilidad y flexibilidad de los balances aproximados son útiles para evaluar la recarga, también se puede utilizar trazadores como herramientas en la determinación del origen de la recarga de los acuíferos.

Los sistemas de flujo del agua subterránea (AS) se ven modificados en el subsuelo de las ciudades debido a la presencia de estructuras enterradas que interactúan con el flujo subterráneo, la infiltración de agua de diferentes fuentes subsuperficiales modifica las condiciones hidrodinámicas (diferencia de presiones y cargas hidráulicas) del AS, así como los elevados índices de explotación presentes en áreas urbanas.

La excesiva explotación del AS es la consecuencia lógica de una densidad poblacional alta o de prácticas y costumbres ineficientes, originando a su vez serios problemas de calidad y cantidad en el agua almacenada en los acuíferos. Los principales problemas son: descenso y ascenso del nivel freático (NF) a lo largo del tiempo, subsidencia del terreno e intrusión de aguas de mar en zonas costeras.

El NF puede descender drásticamente si se producen explotaciones no controladas, minando el recurso y abatiendo la superficie de la zona saturada pudiendo llegar a invertir la dirección de flujo regional, lo cual produce un cambio en la presión de poro del subsuelo reduciendo así su capacidad de resistir cargas verticales.

El análisis de la contaminación del AS en zonas urbanas es muy complicado debido al gran número de potenciales fuentes y la gran variedad de contaminantes que se puede encontrar en las ciudades. Los principales contaminantes son: aguas residuales, fertilizantes y pesticidas, compuestos volátiles orgánicos, hidrocarburos y sus derivados, metales pesados y compuestos especiales, entre otros.

Las fuentes más comunes de contaminantes son: derrames en sitios industriales, disposición y almacenamiento de vertidos, áreas de cultivo, jardines e invernaderos; fugas del sistema de alcantarillado sanitario, pozos negros y tanques sépticos; derrames durante el transporte de compuestos químicos y desechos hospitalarios y farmacéuticos.

La mayor parte de los contaminantes se vierten en la superficie o sub-superficie, por medio de procesos de infiltración y percolación los contaminantes alcanzan la zona saturada contaminando el AS mediante diferentes procesos y reacciones físico-químicas, como son: precipitación, disolución, adsorción, disociación y oxido-reducción, entre otros. Esto se debe a la naturaleza (orgánica, inorgánica) de la gran variedad de contaminantes.

En todas las ciudades, la mayoría de los procesos antes descritos se presentan de forma conjunta y simultánea debido a que están indivisiblemente relacionados, ocasionando sinergia de efectos e impactos ambientales variados, motivo por el cual la gestión ambiental y del agua deben tener un enfoque integral.

En la problemática de los casos de México, no se consideraron durante la planeación y desarrollo de los centros urbanos los escenarios futuros de su desarrollo. Los conceptos, metodologías y experiencias sobre medidas de prevención y mitigación de impactos relacionados al AS, deben ser analizados aplicando la hidrogeología urbana como parte integral de la gestión ambiental en áreas urbanas.

Una gestión inteligente debe tener claro que el AS es un recurso limitado, por tanto debe indagarse su aprovechamiento sostenible. El objetivo de la gestión debe ser proteger, utilizar, reciclar y verter el agua en condiciones ambientalmente satisfactorias, procurando su disponibilidad a las futuras generaciones, además debe involucrar políticas públicas, decisiones sociales y tecnologías limpias.

Las prácticas de gestión desarrolladas hasta ahora son: 
reducción de la extracción, reciclaje de aguas residuales, subsidio para la optimización de sistemas de riego, determinación de la vulnerabilidad de acuíferos, limitación de áreas con alta densidad de viviendas, control de ubicación de tiraderos y rellenos sanitarios, así como su monitoreo y gestión. Se debe procurar restringir el vertido de efluentes industriales directamente sobre cursos de agua, se tienen que introducir medidas especiales en el manejo de compuestos químicos y sus efluentes, deben mejorarse los sistemas planificación de la red de saneamiento y la ubicación de las plantas de tratamiento de aguas residuales y muchos otros, dependiendo del caso de estudio.

El uso de modelos integrales de gestión del agua en las urbes debe ser impulsado e implementado en la mayoría de las grandes ciudades y ciudades con desarrollo económico acelerado, con el objetivo de gestionar ambientalmente el recurso "agua subterránea" en el ciclo hídrico urbano.

\section{Conclusiones}

Debido a la urgente necesidad de conservar, proteger y gestionar el agua subterránea en áreas urbanas, así como brindar soluciones adecuadas a diversos problemas hídricos, emerge una nueva rama científica denominada "hidrogeología urbana", la cual se desprende de la hidrogeología tradicional.

La hidrogeología urbana, es capaz de explicar el comportamiento hidrodinámico de los acuíferos en las peculiares características de las áreas urbanas y en su diversidad de situaciones e interrelaciones entre las aguas subterráneas y el conjunto de edificaciones, espacios, vías de comunicación, dispositivos y servicios que conforman una ciudad. El agua subterránea en zonas urbanas tiene gran interrelación con el desarrollo socioeconómico y urbanístico de la ciudad y requiere de un marco integrado que implica el uso de modelos específicos que puedan ser entendidos por autoridades y planificadores urbanos. Debe reconocerse que el agua subterránea es un componente clave en el proceso de planeación y desarrollo urbano sostenible.

Queda claro que el uso de modelos como el UVQ es muy importante para gestionar los recursos hídricos de una ciudad. Para prevenir la contaminación y viabilizar el uso sostenible, permitiendo fácil acceso y visualización de los datos que nos permitae valuar los impactos ambientales con el objetivo de realizar ajustes en tiempo real, visualizar, interpretar y analizar escenarios futuros.

Los estudios hidrogeológicos urbanos y la implementación de las políticas ambientales en los países en desarrollo están plenamente justificados. Actualmente estos análisis se encuentran en una etapa relativamente incipiente. Los países en desarrollo deben priorizar temas económicos y ambientales para garantizar un desarrollo sostenible en muchas de sus importantes ciudades.

Las ciudades importantes, en países con escasos recursos hídricos como México, dependen cada vez más del aprovechamiento del agua subterránea para viabilizar su crecimiento, por tanto la hidrogeología urbana es una ciencia clave y prioritaria para su desarrollo sostenible en estos países y alrededor del mundo.

Se ha revisado el estado actual de la hidrogeología urbana, desde su parte conceptual, su desarrollo y aplicación. Se realizó el análisis de los casos de estudio más significativos del mundo y de México. A futuro deberán desarrollarse y estudiarse de manera particular aún más casos con la finalidad de desarrollar las capacidades de la hidrogeología urbana como ciencia de vital importancia en el uso, manejo, preservación y contaminación de los recursos hídricos de las ciudades desde un enfoque integral.

\section{Referencias}

Abidin, H.Z., Andreas, H., Djaja, R., Darmawan, D., Gamal, M., 2008, Land subsidence characteristics of Jacarta between 1997 and 2005, as estimated using GPS surveys: GPS Solutions, 12, 23-32.

Adrian, O.G., Rudolph, D.L., Cherry, J.A., 1999, The analysis of long term land subsidence near Mexico City: field investigations and predictive modeling: Water Resources Research, 35, 3327-3341.

Aichi, M., 2008, Coupled Groundwater Flow/Deformation Modelling for Predicting Land Subsidence, en Takizawa, S. (ed.), Groundwater Management in Asian Cities: Technology and Policy for Sustainability: Tokio, Springer, 105-124.

Al-Sefry, S.A., Sen, Z., 2006, Groundwater rise problem and risk evaluation in major cities of arid lands - Jedddah Case in Kingdom of Saudi Arabia: Water Resources Management, 20, 91-108.

Amelung, F., Galloway, D.L., Bell, J.W., Zebker, H.A., Laczniak, R.J., 1999, Sensing the ups and downs of Las Vegas: InSAR reveals structural control of land subsidence and aquifer-system deformation: Geology, 27, 483-486.

Ames, D.P., 2002, Bayesian decision network for watershed management: Logan, Utah, EE.UU., Utah State University, tesis doctoral, 250 p.

Apodaca, L.E., Bails, J.B., Smith, C.M., 2002, Water quality in shallow alluvial aquifers, Upper Colorado River Basin, Colorado, 1997: Journal of the American Water Resources Association, 38, 133-149.

Arzate-Flores, J.A., Pacheco-Martínez, J., Del Rosal-Pardo, A., BarbozaGudiño, R., Mata-Segura, J.L., López-Doncel, R., 2006, Carta de riesgo de agrietamientos del área metropolitana de San Luis Potosí: Geos, 26, 185.

Atabey, E., 2005, Tibbi Jeoloji, en Atabey, E. (ed.), TMMOB Jeoloji Mühendisleri Odasi Yayinlari: Ankara, TMMOB, 194.

Ator, S.W., Denis, J.M., 1997, Relation of nitrogen and phosphorus in ground water to land use in four subunits of the Potomac River Basin: Baltimore, Maryland, United States Geological Survey, Water-Resources Investigations Report, 97-4268, 26 p.

Ávila-Olivera, J.A., Garduño-Monroy, V.H., 2006, El abatimiento de los niveles fréaticos es sólo un elemento en los procesos de subsidenciacreep-falla, caso: la ciudad de Morelia, Michoacán: Geos, 26, 186.

Ávila-Olivera, J.A., Farina, P., Garduño-Monroy, V.H., 2006, Aplicación de la técnica DinSAR en el estudio de los procesos de subsidenciacreep-falla en la ciudad de Morelia, Michoacán: Geos, 26, 184.

Bassington, F.C., 1990, Rising groundwater levels in the United Kingdom: Proceedings of the Institution of Civil Engineers, 88, 1037-1057.

Bitelli, G., Bonsignore, F., Unguendoli, M., 2000, Levelling and GPS networks to monitor ground subsidence in the Southern Po Valley: Journal of Geodinamics 30, 355-369.

Bucich, N.G., Nagy, M.I., 2003, Affectation of urban structures by variation of phreatic level (natural and anthropic), Buenos Aires, Argentina: RMZ - Materials and Geoenvironment, 50, 79-82.

Burston, M.W., Nazari, M.M., Bishop, P.K., Lerner, D.N., 1993, Pollution of groundwater in the Coventry region (UK) by chlorinated hydrocarbon solvents: Journal of Hydrology, 149, 137-161. 
Cabral-Cano, E., Díaz-Molina, O., 2006, El gradiente de subsidencia, una herramienta para la determinar el potencial fracturamiento superficial en la ciudad de México mediante InSAR: Geos, 26, 184.

Comisión de Agua Potable y Drenaje de Monterrey (CAPDM), 1990, Proyecto Monterrey IV: Monterrey, Comisión de Agua Potable y Drenaje de Monterrey, $33 \mathrm{p}$.

Carbognin, L., Gatto, P., Mozzi, G., 1984, Venice subsidence case history, en Poland, J.F: (ed.), Guidebook to studies of land subsidence due to ground-water withdrawal: París, UNESCO, 161-174.

Carreón-Freyre, D., Cerca, M., Luna-González, L., Gámez-González, F.J., 2005, Influencia de la estratigrafía y estructura geológica en el flujo de agua subterránea del Valle de Querétaro: Revista Mexicana de Ciencias Geológicas, 22, 1-18.

Clifforde, I.T., Tomicic, B., Mark, O., 1999, Integrated water management, a Europe vision for the future, en Proceedings of the $9^{\text {th }}$ internationalconference on urban storm Drainage: Portland, Oregon, EE.UU, American Society of Civil Engineers, 8-13.

Comisión Nacional del Agua (CNA), 2007, Estadísticas del Agua en México: México D.F., Comisón Nacional del Agua, 259 p.

Cook, A.M., Scholtz, R., Leisinger, T., 1988, Mikrobieller Abbau von halogenierten aliphatischen Verbindungen (Microbial degradation of halogenated aliphatic compounds): GWF Wasser/Abwässer, $129,61-68$.

Custodio, E., 2002, Aquifer overexploitation: what does it mean?: Hydrogeology Journal, 10, 254-277.

Custodio, E., 2004, Hidrogeología Urbana: una nueva rama de la ciencia hidrogeológica: Boletín Geológico y Minero, 115, 283-288.

Chilton, J.E., 1999, Groundwater in the urban environment: Selected city profiles : Rotterdam, Balkema, $356 \mathrm{p}$.

Danis, M., 2005, Ithalat ve ithalatin dentimi, agir metal kirlilikleri (Import and control of import, heavy metal pollution), en Workshop of fertilizer and fertilizer raw materials: Diyarbakir, Publications of Chamber of Geology Engineers of Turkey TMMOB, 25-27.

de León-Gómez, H., Schetelig, K., Meiburg, P., 1994, Abastecimiento de agua potable en el noreste de México ejemplo Presa Cerro Prieto: Zentralblatt für Geologie und Paläontologie, I, 593-602.

Declercq, P.Y., Devleeschouwer, X., Pouriel, F., 2005, Subsidence revealed by PSInSAR technique in the Ottignies-Wavre area (Belgium) related to water pumping in urban area (expanded Abstract), en Fringe 2005 Workshop: Frascati, Italia, European Space Agency, 6 p.

Dehls, J.F., Basilico, M., Colesanti, C., 2002, Ground deformation monitoring in the Ranfjord area of Norway by means of the permanentscatterers technique, en $23^{\text {th }}$ International Geoscience and Remote Sensing Symposium: Toronto, Institute of Electrical and Electronics Engineers, 203-207.

Downing, R.A., 1993, Groundwater resources, their development and management in the UK: an historical perspective: Quarterly Journal of Engineering Geology and Hydrogeology, 26, 335-358.

Drewes, J.E., Heberer, T., Rauch, T., Reddersen, K., 2003, Fate of pharmaceuticals during ground water recharge: Ground Water Monitoring \& Remediation 23, 64-72.

Ekemen-Keskin, T., 2010, Nitrate and heavy metal pollution resulting from agricultural activity: a case study from Eskipazar (Karabuk, Turkey): Enviromental Earth Sciences, 61, 703-721.

Elhatip, H., Afsin, M., Kuscu, I., Dirik, K., Kurmac, Y., Kavurmaci, M., 2003, Influences of human activities and agriculture on groundwater quality of Kayseri-Incesu-dokuzpinar springs, central Anatolian part of Turkey: Environmental Geology, 44, 490-494.

Ellis, P.A., Rivett, M.O., 2007, Assessing the impact of VOC-contaminated groundwater on surface water at the city scale: Journal of Contaminant Hydrology, 91, 107-127.

Escolero, O., Torres-Onofre, S., 2007, Análisis de la intrusión de agua de mar en el acuífero de La Paz (México): Boletín Geológico y Minero, 118, 637-648.

Escolero, O.A., Marin, L.E., Steinich, B., Pacheco, J., 2000, Delimitation of a hydrological reserve for a city within a karstic aquifer: the Merida, Yucatan example: Landscape and Urban Planning, 51, 53-62.

Escolero, O.A., Marin, L.E., Steinich, B., Pacheco, A.J., Cabrera, S.A., Alcocer, J., 2002, Development of a Protection Strategy of Karst
Limestone Aquifers: The Merida Yucatan, Mexico Case Study: Water Resources Management, 16, 351-367.

Ferreti, A., Prati, C., Rocca, F., 2000, Nonlinear subsidence rate estimation using permanent scatterers in differential SAR interferometry: IEEE Transactions on Geoscience and Remote Sensing, 38, 2202-2212.

Ferreti, A., Prati, C., Rocca, F., 2001, Permanent scatterers in SAR interferometry: IEEE Transactions on Geoscience and Remote Sensing, 39, 8-20.

Fetter, C.W., 2001, Applied Hydrogeology: Englewood Cliffs, New Jersey, EE.UU., Prentice Hall, 598 p.

Ford, M., Tellam, J.H., 1994, Source, type and extent of inorganic contamination within the Birmingham aquifer system: Journal of Hydrology, 156, 101-135.

Foster, S., Lawrence, A., Morris, B., 2001, Las aguas subterráneas en el desarrollo urbano: Evaluación de las necesidades de gestión y formulación de estrategias: Washington D.C., Banco Internacional de Reconstruccion y Fomento/Banco Mundial, $60 \mathrm{p}$.

Foster, S.S.D., Chilton, P.J., 2004, Downstream of downtown: urban wastewater as groundwater recharge: Hydrogeology Journal, 12, 115-120.

Freeze, R.A., Cherry, J.A., 1979, Groundwater: Englewood Cliffs, New Jersey, EE.UU., Prentice Hall, 604 p.

Fuge, R., 2004, Anthropogenic sources, en Selinus, O., Alloway, B.J., Centeno, J.A., Finkelman, R.B., Fuge, R., Lindh, U., Smedley, P. (eds.), Essential of medical geology, impact of the natural environment on public health: San Diego, California, ElsevierAcademic Press, 43-60.

Gambolati, D., Frezze, R.A., 1973, Mathematical simulation of the subsidence of Venice: theory: Water Resources Research, 9, 721-733.

Grischek, T., Nestler, W., Piechniczek, D., Fischer, T., 1996, Urban groundwater in Dresden, Germany: Hydrogeology Journal, 4, 48-63.

Guo, F., Jiang, G., Yuan, D., 2007, Major ions in typical subterranean rivers and their anthropogenic impacts in southwest karst areas, China: Environmental Geology, 53, 533-541.

Hayashi, T., 2008, The Shallow Groundwater Environment and the Risk of Soil Liquefaction, en Takizawa, S. (ed.), Groundwater Management in Asian Cities: Technology and Policy for Sustainability: Tokio, Springer, 81-103.

He, Z.L., Yang, X.E., Stoffella, P.J., 2005, Trace elements in agroecosystems and impacts on the environment: Journal of Trace Elements in Medicine and Biology, 19, 125-140.

Hooker, P.J., McBridge, D., Brown, M.J., Lawrence, A.R., Gooddy, D.C., 1999, An integrated hydrogeological case study of a postindustrial city in the West Midlands of England, en Chilton, J. (ed.), Groundwater in the Urban Environment: Selected City Profiles: Rotterdam, Países Bajos, Balkema, 145-150.

Instituto Nacional de Geografía Estadística e Informática (INEGI), 2005, Segundo Conteo de Población y Vivienda: Proyecto IRIS-Science II (CD-ROM): Aguascalientes, Aguascalientes, Instituto Nacional de Estadística, Geografía e Informática, 1 CD-ROM.

Johnson, S.T., 1994, Rising groundwater levels: engineering and environmental implications, en Wilkinson, W.B. (ed.), Groundwater Problems in Urban Areas: Londres, Thomas Telford, 285-298.

Kessler, A., Rubin, H., 1987, Relationships between water infiltration and oil spill migration in sandy soils: Journal of Hydrology, 91, 187-204.

Kim, J.S., Kim, D.J., Kim, S.W., Won, J.S., Moon, W.M., 2007, Monitoring of urban land surface subsidence using PSInSAR: Geoscience Journal, 11, 59-73.

Kim, S.W., 2003, Measurement of surface displacement of Mt. Baekdu and Busan area using L-band SAR interferometry: Seúl, Corea del Sur, Yonsei University, tesis doctoral, $157 \mathrm{p}$.

Kontogianni, V., Pytharouli, S., Stiros, S., 2007, Ground subsidence, Quaternary faults and vulnerability of utilities and transportation networks in Thessaly, Greece: Environmental Geology, 52, 10851095.

Krishna, A.K., Govil, P.K., 2004, Heavy metal contamination of soil around Pali Industrial Area, Rajasthan, India: Environmental Geology, 47, 38-44. 
Kropp, W.W., 2010, A Spatial Multicriteria Decision Analysis Approach for Evaluating Sustainable Development: Athens, Ohio, EE.UU., Ohio University, tesis de maestría, 125 p.

Kuczera, G., Coombes, P.J., 2002, Towards continuous simulation: a comparative assessment of flood performance of volume-sensitive systems, en SIA 2002 conference of stormwater management: Orange, New South Wales, Australia, Stormwater Industry Association.

Kumbur, H., Özsoy, H.D., Özer, Z., 2008, Mersin Il'nde tarimsal alanlarda kullanilan kimyasallarin su kalitesi üzerine etkilerinin berlinlenmesi (Determination of the effects of chemical used in agricultural area on water quality in Mersin Province): Cev-Kor Ekoloji, 68, 54-58.

Kuroda, K., Fukushi, T., 2008, Groundwater Contamination in Urban Areas, en Takizawa, S. (ed.), Groundwater Management in Asian Cities: Technology and Policy for Sustainability: Tokio, Springer, 126-149.

Larson, K.J., Basagaoglu, H., Mariño, M.A., 2001, Prediction of optimal safe ground water yield and land subsidence in the Los BanosKettleman City area, California, using a calibrated numerical simulation model: Journal of Hydrology, 242, 79-102.

Leakea, S.A., Mock, P.A., 1997, Dimensionality of ground water flow models: Ground Water, 35, 930.

Lekkas, D.F., Manoli, E., Assimacopoulos, D., 2008, Integrated urban water modeling using the Aquacycle model: Global NEST Journal, 10, 310-319.

Lerner, D.N., 2004, Urban Groundwater Pollution: Lisse, Países Bajos, Balkema, 243 p.

Lerner, D.N., Barrett, M.H., 1996, Urban groundwater issues in the United Kingdom: Hydrogeology Journal, 4, 80-89.

Lerner, D.N., Halliday, D., Hoffman, J.M., 1994, The impact of sewers on groundwater quality, en Wilkinson, W.B. (ed.), Groundwater Problems in Urban Areas: Londres, Thomas Telford, 64-75.

Lobo-Guerrero Uscátegui, A., 2003, Effects of aquifer overexploitation on the surface infraestructure in the Bogotá Sabana (Colombia): RMZ - Materials and Geoenvironment, 50, 193-196.

Lofgren, B.L., 1969, Field measurements of aquifer-system compaction, the San Joaquin Valley, California, en Tison, L.J. (ed.), Land Subsidence, Vol. 1: Lovaina, Bélgica, International Association of Scientific Hydrology, 272-284.

Llamas, M.R., Custodio, E., 2003, Intensive use of groundwater: challenges and opportunities: Lisse, Países Bajos, Balkema, 478 p.

Lloyd, J.W., Heathcote, J.A., 1985, Natural Inorganic Hydrochemistry in Relation to Ground-Water: Nueva York, Oxford University Press, $250 \mathrm{p}$.

Manta, D.S., Angelone, M., Bellanca, A., Neri, R., Sprovein, M., 2002, Heavy metals in urban soils: a case study from the city of Palermo (Sicily), Italy: The Science of The Total Environment, 300, 229-243.

Martínez, S.E., Escolero, O., Wolf, L., 2011, Total Urban Water Cycle Models in Semiarid Environments - Quantitative Scenario Analysis at the Area of San Luis Potosi, Mexico: Water Resources Management, 25, 239-263.

Massonet, D., Feigl, K.L., 1998, Radar interferometry and its application to changes in the Earth's surface: Reviews of Geophysics, 36, 441-500.

McBride, M.B., 2004, Molybdenum, sulfur, and other trace elements in farm soils and forages after sewage sludge application: Communications in Soil Science and Plant Analysis, 35, 517-535.

Mikayilov, F.D., Acar, B., 1998, Toprak ekosistemlerinde kirleticilerin tasinim mekanizmasinin incelenmesi ve modellenmesi (Investigation and modelling of contaminant's movement processes within the soil ecosystems): Ekoloji, 28, 20-23.

Mitchell, V.G., McMahon, T.A., Mein, R.G., 2003, Components of the total water balance of an urban catchment: Environmental Management, 32, 735-746.

Molina, J.S., García-Aróstegui, J.L., Benavente, J., Varela, C., de la Hera, A., López-Geta, J.A., 2009, Aquifers overexploitation in SE Spain: A proposal for the integrated analysis of water management: Water Resources Management, 23, 2737-2760.

Moon, W.M., Ristau, J., Vachon, P., 1998, Feasibility of applying spaceborne SAR interferometry for earthquake tectonic investigation:
Geoscience Journal, 2, 78-87.

Morris, B.L., Darling, W.G., Cronin, A.A., Rueedi, J., Whitehead, E.J., Gooddy, D.C., 2006, Assessing the impact of modern recharge on a sandstone aquifer beneath a suburb of Doncaster, UK: Hydrogeology Journal, 14, 979-997.

Nagarajan, R., Rajmohan, N., Mahendran, U., Senthamilkumar, S., 2010, Evaluation of groundwater quality and its suitability for drinking and agricultural use in Thanjavur city, Tamil Nadu, India: Environmental Monitoring and Assessment, 171, 289-308.

Ng, A.H.M., Chang, H.C., Zhang, K., Ge, L., Rizos, C., 2009, Land subsidence monitoring in Australia and China using Satellite Interferometry, en Sideris, M.G. (ed.), Observing our Changing Earth: Proceedings of the 2007 IAG General Assembly, Perugia, Italy, July 2 - 13, 2007: Berlin/Heidelberg, Springer, 743-750.

Nouri, J., Mahvi, A.H., Babaei, A.A., Jahed, G.R., Ahmadpour, E., 2006, Investigation of heavy metals in groundwater: Pakistan Journal of Biological Sciences, 9, 377-384.

O'Shea, M.J., 1993, Discussion on rising groundwater levels, en Wilkinson, W.B. (ed.), Groundwater Problems in Urban Areas: Londres, Thomas Telford, 363-364.

Ochoa-González, G.H., Carreón-Freyre, D.C., 2006, Influencia de la variación de las propiedades hidráulicas del medio geológico en el análisis de deformación diferencial: Geos, 26, 183.

Organización de las Naciones Unidas para la Educación, la Ciencia y la Cultura (UNESCO), 2006, Unesco Water e-Newsletter No. 161: Water-Related Diseases (documento en línea): París, Organización de las Naciones Unidas para la Educación, la Ciencia y la Cultura, publicado 26 de octubre de 2006, disponible en <www.unesco.org/ water/news/newsletter/161.shtml $>$, consultado 22 de junio de 2011.

Pacheco-Martínez, J., Arzate-Flores, J.A., Barboza-Gudiño, R., LópezDoncel, R., Mata-Segura, J.L., Del Rosal-Pardo, A., 2006, Carta de hundimientos del área metropolitana de San Luis Potosí a partir de un modelo de subsidencia basado en el concepto de esfuezos efectivos: Geos, 26, 185 .

Pacheco, J., Marin, L., Cabrera, A., Steinich, B., Escolero, O., 2001, Nitrate temporal and spatial patterns in 12 water-supply wells, Yucatan, Mexico: Environmental Geology, 40, 708-715.

Panchow, J.F., Cherry, J.A., 1996, Dense chlorinated solvents and other DNAPLs in groundwater: history, behavior, and remediation: Portland, Oregon, Waterloo Press, 522 p.

Phien-wej, N., Giao, P.H., Nutalaya, P., 2006, Land subsidence in Bangkok, Thailand: Engineering Geology, 82, 187-201.

Poland, J.F., 1982, Guidebook to studies of land subsidence due to groundwater withdrawal: París, UNESCO, 305 p.

Rahnama, M.B., Moafi, H., 2009, Investigation of land subsidence due to groundwater withdraw in Rafsanjan plain using GIS software: Arabian Journal of Geosciences, 2, 241-246.

Rajmohan, N., Al-Futaisi, A., Al-Touqi, S., 2009, Geochemical process regulating groundwater quality in a coastal region with complex contamination sources: Barka, sultanate of Oman: Environmental Earth Sciences, 59, 385-398.

Raymond, D., Rudant, J.P., 1997, ERS-1 SAR interferometry: potential and limits for mining subsidence detection, en $3^{\text {rd }}$ ERS Symposium on Space at the Service of our Environment: Florencia, Italia, European Space Agency, 541-544.

Reynolds, J.H., Barrett, M.H., 2003, A review of the effects of sewer leakage on groundwater quality: Water and Environment Journal, 17, 34-39.

Ribeiro, L., 2002, Future Groundwater Resources at Risk: Proceedings of the $3^{\text {rd }}$ International Conference, Lisbon, Portugal, June 2001: Lisboa, CVRM - Centro de Geo-Sistemas 753 p.

Schellekens, G.A.P., 1974, Olieverontreiniging pompstation Geulhen: $\mathrm{H}_{2} 0,7,140-143$.

Schirmer, M., Butler, B.J., 2004, Transport behaviour and natural attenuation of organic contaminants at spill sites: Toxicology, 205, 173-179.

Schwille, F., 1975, Groundwater pollution by mineral oil products en Groundwater Pollution Symposium, 1971: Moscú, International Association of Hydrological Sciences, 226-240. 
Shanahan, P., 2009, Groundwater in the urban environment, en Baker, L.A. (ed.), The Water Environment of Cities: Nueva York, Springer, 29-48.

Shen, Y., 2008, Groundwater Resources in Urban Water Management, en Takizawa, S. (ed.), Groundwater Management in Asian Cities: Technology and Policy for Sustainability: Tokio, Springer, 35-59.

Short, C.S., 1988, The Bramham incident, 1980 - An outbreak of water borne infection: Water \& Environment Journal, 2, 383-390.

Solomon, S., Qin, D., Manning, M., Chen, Z., Marquis, M., Averyt, K.B., Tignor, M., Miller, H., 2007, Contribution of Working Group I to the fourth assessment report of the intergovernmental panel on Climate Change, 2007: Nueva York, Cambridge University Press, 996 p.

Stenger, D.B., O'Reilly, S., O'Halloran, J., 2006, Contaminants and pollutants, en Davenport, J., Davenport, J.L. (eds.), The ecology of transportation: managing, mobility for the environment: Dordrecht, Países Bajos, Springer, 361-389.

Takizawa, S., 2008a, Groundwater use and mangement in urban areas, en Takizawa, S. (ed.), Groundwater management in Asian cities: technology and policy for sustainability: Tokio, Springer, 13-33.

Takizawa, S., 2008b, Introduction, en Takizawa, S. (ed.), Groundwater management in Asian cities: technology and policy for sustainability: Tokio, Springer, 3-11.

Toussaint, B., 1994, Umweltproblematik und Hydrogeologie der Erkundung von Böden- und Grundwasser-Kontaminationen durch leichtflüchtige halogenierte Kohlenwasserstoffe (Environmental problems and hydrogeology of investigation of soil and groundwater contamination by halogenated organic volatiles): Wiesbaden, Alemania, Hessische Landesanstalt für Umwelt und Geologie, 327 p.

Vanlooke, R., DeBorger, R., Voets, J.P., Verstraete, W., 1975, Soil and groundwater contamination by oil spills-problems and remedies: International Journal of Environmental Studies, 8, 99-111.

Vázquez-Suñé, E., Sánchez-Vila, X., Carrera, J., 1999, Gestión de las aguas subterráneas en zonas urbanas, conceptualización y modelación: aplicación a Barcelona (España), en Tineo, A. (ed.), Hidrología Subterránea: Tucumán, Argentina, Instituto Superior de Correlación Geológica, 153-160.

Vázquez-Suñé, E., Sánchez-Vila, X., Carrera, J., 2005, Introductory review of specific factors influencing urban groundwater, an emerging branch of hydrogeology, with reference to Barcelona, Spain: Hydrogeology Journal, 13, 522-533.
Welty, C., 2009, The urban water budget, en Baker, L.A. (ed.), The water environment of cities: Nueva York, Springer, 17-27.

Wilkinson, W.B., 1994, Groundwater problems in urban areas: Londres, Thomas Telford, $453 \mathrm{p}$.

Wolf, L., Klinger, J., Hötzl, H., Mohrlok, U., 2007, Quantifying mass fluxes for urban drainage systems to the urban soil-aquifer system: Journal of Soils and Sediments, 7, 85-95.

Wolf, L., Morris, B., Dillon, P., Vanderzalm, J., Rueedi, J., Burns, S., Cook, S., 2006, AISUWRS urban water resources toolbox - a brief summary, en Wolf, L., Morris, B., Burns, S. (eds.), Urban water resources toolbox: integrating groundwater into urban water management: Londres, International Water Association, 282-290.

Wong, T., Coleman, J., Duncan, H., Fletcher, T., Jenkins, G., Siriwardena, L., Wotton, R., 2002, Music model for urban stormwater improvement conceptualization (programa informático): Canberra, Australia, eWater CRC, disponible en <www.toolkit.net.au/Tools/MUSIC/ documentation>, consultado 30 de octubre de 2010 .

Wright, P., Stow, R., 1999, Detecting mining subsidence from space: International Journal of Remote Sensing, 20, 1183-1188.

Xiaoqin, W., 2009, A proposal and application of the integrated benefit assessment model for urban water resources exploitation and utilization: Water Resources Management, 23, 1171-1182.

Zhang, A.G., Wei, Z.X., 2005, Land subsidence in China: Shanghai, China, Science and Technology Press, $240 \mathrm{p}$.

Zhang, Y., Xue, Y.Q., Wu, J.C., Shi, X.Q., Yu, J., 2010, Excessive groundwater withdrawal and resultant land subsidence in the $\mathrm{Su}-\mathrm{Xi}$ Chang area, China: Environmental Earth Sciences, 61, 1135-1143.

Manuscrito recibido: Diciembre 5, 2010.

Manuscrito corregido recibido: Mayo 7, 2011.

Manuscrito aceptado: Mayo 14, 2011. 